Angelique Haugerud

\title{
The Culture of Politics in Modern Kenya
}

Cambridge University Press, Cambridge, 1995, 266 S., $£ 35.00$

Die US-amerikanische Anthropologin Angelique Haugerud beschäftigt sich in der vorliegenden Studie mit einem bisher in der Afrika-Wissenschaft vernachlässigten Gebiet: der politischen Kultur. Am Beispiel Kenya demonstriert die Autorin, wie die seit 1990 praktizierte Politik der westlichen Geberstaaten, die Fortsetzung der Entwicklungshilfe von der Einführung demokratischer Reformen abhängig zu machen, sich bisher auf die innerstaatliche politische Kultur auswirkte. In Kenya gab es eine lange Tradition von Opposition, die immer wieder in unterschiedlichen Formen, wie z.B. der Anprangerung von Korruption, in Erscheinung trat. Die politischen Auseinandersetzungen zwischen den Anhängern des Einparteien- und des Mehrparteiensystems gehen im wesentlichen auf diese historischen Differenzen zurück. Ein Teil der Mehrparteienanhänger sah die Demokratisierung nicht als Selbstzweck, sondern vielmehr als Vehikel an, um die eigenen Interessen, die unter dem autoritären Regime unterdrückt wurden, durchzusetzen. Die Oppositionsbewegung gegen das Regime von Daniel arap Moi wurde von verschiedenen Gruppen getragen (Akademikern, Geistlichen und einem Teil des Unternehmertums), die sich von der herrschenden Staatsklasse in ihren Entfaltungsmöglichkeiten eingeengt sahen.

Bemerkenswert ist, welche Rolle die Musik in einem Staat einnahm, in dem die Medien staatlicher Kontrolle unterlagen. Die regimekritischen Musikcassetten, die im informellen Straßenhandel kursierten, wurden besonders gern von privaten Minibus- und Sammeltaxibetreibern abgespielt. Sie bildeten eine alternative Informationsquelle über politische Ereignisse. Eine behördliche Anordnung, mit der das Abspielen von Musik im Straßenverkehr ganz verboten werden sollte, war nicht durchzusetzen und blieb somit wirkungslos. Während die subversive Musik auch in ländlichen Gegenden wahrgenommen wurde, richteten sich Theaterstücke, mit denen indirekt das Regime der Lächerlichkeit preisgegeben wurde, vor allem an die Mitglieder der urbanen Mittelschicht.

Von besonderem Interesse sind die Erkenntnisse, die auf Feldforschungsergebnissen basieren, die die Autorin von 1978 bis 1981 im Embu District durchgeführt hat (Kapitel 3). Haugerud analysiert den Ablauf öffentlicher Kundgebungen, sogenannte "baraza", auf denen lokale und nationale Politiker und Verwaltungsbeamte die Teilnehmer in den politischen Entscheidungsprozeß einzubinden versuchten. Obwohl es sich dabei nicht um einen wirklichen Dialog zwischen den Vertretern des Staates und den ländlichen Teilnehmern handelte, sondern in erster Linie um ein Akklamationsforum für staatliche Beschlüsse, konnte spontan durchaus Kritik an einzelnen - meist lokalen - Mißständen geübt werden. Die Veranstaltung war dennoch nicht ohne Risiko: Der Redner mußte durch rhetorisches Geschick versuchen, die Teilnehmer zu manipulieren, indem ihnen die Notwendigkeit einer eigentlich unpopulären Regierungsentscheidung so lange suggeriert wurde, bis sie diese unterstützten. Die Autorin demonstriert diesen Aspekt am Verbot des traditionellen häuslichen Bierbrauens, das offiziell mit der Bekämpfung der Trunksucht begründet wurde, in 
Wirklichkeit aber die heimische Bierindustrie und damit die privaten wirtschaftlichen Interessen der Regimemitglieder stärken sollte. Häufig wurde "baraza" auch als Selbstdarstellungsinstrument genutzt, indem der Redner indirekt politische Konkurrenten kritisierte oder auf die Medienwirksamkeit seines Auftrittes abzielte. Durch die permanente Interaktion zwischen dem Redner, der über Gestaltungsfreiräume verfügte, und dem Publikum war der Verlauf der Veranstaltung zwar zum größten Teil, aber nicht vollständig berechenbar.

Die Autorin arbeitet die Aufgaben der "baraza" deutlich heraus: Erstens wurde mit diesen Kundgebungen die Präsenz des Regimes symbolisch dargestellt, zweitens die Regierung zu legitimieren gesucht, und drittens konnte auf diesem Wege die politische Debatte vom Regime kontrolliert und begrenzt werden.

Als das Moi-Regime Anfang der 1990er Jahre unter Demokratisierungsdruck geriet, wurde "baraza" verstärkt für Propaganda gegen die Einführung demokratischer Reformen genutzt, indem ein Mehrparteiensystem mit Tribalismus und Bürgerkrieg identifiziert wurde.

Haugerud untersucht in einem historischen Abriß (ab 1890) auch die Patron-Klient-Beziehungen, die in Kenya eine wichtige Rolle gespielt haben und noch heute spielen. Besonders in Zeiten der Nahrungsmittelknappheit gerieten diese Beziehungen unter Druck, da der Rhetorik, die während der "baraza" gepflegt wurde, nun Taten folgen mußten. Dem entging z.B. das Moi-Regime, indem es sowohl an die von der Hungerkrise nicht Betroffenen als auch an die internationale Gemeinschaft appellierte, den Hungernden zu helfen. Der moralischen Aufgabe des Regimes war damit Genüge getan. Die Autorin analysiert die PatronKlient-Beziehungen anhand eigener Feldforschungsergebnisse im Embu District. Sie geht dabei auf verschiedene Verhaltens- und Konfliktlinien ein: Verhältnis zwischen den Generationen, zwischen Ehepartnern, den Ehefrauen untereinander, zwischen Reichen und Armen.

Die Studie bietet interessante Einblicke in die politische Kultur Kenyas. Nicht ganz gelungen ist die Gliederung. Sie würde eher überzeugen, wenn das Kapitel über die Demokratisierungsphase ab 1990 statt an den Anfang an das Ende der Studie gesetzt worden wäre. Die Erkenntnisse aus den zeitlich vorgeschalteten Themenkapiteln hätten dann besser zur Darstellung der Bedeutung von politischer Kultur im Demokratisierungsprozeß herangezogen werden können.

Heiko Meinhardt 